PUPT-1477, June 1994

\title{
Renormalization Group Derivation of the Localization Length Exponent in the Integer Quantum Hall Effect
}

\author{
L. Moriconi \\ Physics Department, Princeton University \\ Jadwin Hall, Princeton, NJ 08544, U.S.A.
}

\begin{abstract}
We compute, neglecting possible effects of subleading irrelevant couplings, the localization length exponent in the integer quantum Hall effect, for the case of white noise random potentials. The result obtained is $\nu=2$ for all Landau levels. Our approach consists in a renormalization group transformation of Landau orbitals, which iterates the generating functional of Green's functions for the localization problem. The value of $\nu$ is obtained from the asymptotic form of the renormalization group mapping. The basic assumptions in our derivation are the existence of a scaling law for the localization length and the absence of Landau level mixing.
\end{abstract}




\section{INTRODUCTION}

The observation of the quantum Hall effect [1] was a surprise in the context of the physics of disordered systems. It is puzzling that there must be, from the clear experimental evidence of the Hall conductivity plateaus, delocalized states which transport current, while it is well-known that in disordered two-dimensional systems (in the absence of magnetic fields) all states are localized [2]. The picture which has emerged over the last decade is that near the center of each Landau level, broadened by the presence of disorder, there is a specific value of energy, $E_{c}$, such that states with energy $E$, close enough to $E_{c}$, are characterized by a divergent localization length, given by $\xi\left(E-E_{c}\right) \sim\left|E-E_{c}\right|^{-\nu}$. This result has been supported by analytical, numerical, and experimental studies [3,5, 13]. Aoki and Ando [7] have found numerically $\nu \lesssim 2$ and $\nu \lesssim 4$, in the case of $\delta$-impurities, for the first and second Landau levels, respectively. Subsequent work by many authors confirmed their computation, in the first Landau level, in particular the semiclassical analysis of Mil'nikov and Sokolov [5] giving $\nu=7 / 3$ and the perturbation approach of Hikami [3], which yields $\nu=1.9 \pm 0.2$ (it is worthwhile mentioning, however, that a careful interpretation of Hikami's result, in the light of the multifractal structure of wavefunctions, gives $\nu=2.4 \pm 0.3$, as it was observed in 4 | $)$. Experimentally [13], the value of $\nu$ was found to be close to 2.3. A difficulty in the subject is that, experimentally, Coulomb effects are expected to modify the result obtained when two-body interactions are neglected and, therefore, the good agreement between numerical [9 12 and experimental results is not very well understood. Another question is the precise dependence of $\nu$ with the Landau level index. A recent numerical analysis [12 suggests that for finite range potentials the value of $\nu$ is universal, being the same for all Landau levels.

Our aim in this paper is to show a non-perturbative analysis of the problem, in the case of white noise random potentials. We will assume the existence of a scaling law for $\xi$ and neglect the Landau level mixing, which are the usual starting points in most of approaches. The result we found, taking into account only irrelevant couplings of leading order, is $\nu=2$ for all Landau levels, indicating the universal behaviour of the localization length exponent 
in any transition between plateaus in the integer quantum Hall effect.

The method employed here consists in a redefinition of Landau orbitals, inspired by the block spin renormalization group [14]. While in the case of general Ising models the construction of renormalized Hamiltonians is an approximation, we will carry an analogous approach in our problem, obtaining a sequence of exactly renormalized generating functionals of Green's functions. At each step of the renormalization group iteration, the localization length decreases by a factor $1 / 2$. Examining, then, the fixed point form of the consecutive transformations, we are able to obtain the critical exponent $\nu$.

The plan of the paper is the following. In section II we outline the method, considering the generating functional of averaged advanced and retarded Green's functions and showing how a modification of the localization length is associated to a redefinition of the coupling constants of the system. An analytical relation for $\nu$ is obtained. In section III we define the renormalization group transformation and present a detailed computation of $\nu$. Finally, in section IV, we conclude the analysis and comment on our results.

\section{OUTLINE OF THE METHOD}

The localization length of a general disordered system may be found through a computation of $\xi^{-1}(E)$, defined as

$$
\xi^{-1}(E)=-\frac{1}{2} \lim _{\left|\vec{x}-\vec{x}^{\prime}\right| \rightarrow \infty} \frac{\ln <G_{+}\left(\vec{x}, \vec{x}^{\prime} ; E\right) G_{-}\left(\vec{x}, \vec{x}^{\prime} ; E\right)>}{\left|\vec{x}-\vec{x}^{\prime}\right|}
$$

where the brackets in the above expression mean an average over the realizations of a random potential $V$ and

$$
G_{ \pm}\left(\vec{x}, \vec{x}^{\prime} ; E\right)=<\vec{x}\left|\left(E-H+i 0^{ \pm}\right)^{-1}\right| \vec{x}^{\prime}>
$$

are the retarded and advanced Green's functions associated to the Hamiltonian $H=H_{0}+$ $V$. A pratical way to study the averaged product of Green's functions is considering the generating functional (in two spatial dimensions) 


$$
\begin{aligned}
Z\left[\bar{j}_{1}, j_{1}, \bar{j}_{2}, j_{2}\right] & =\int D \bar{u}_{1} D u_{1} D \bar{u}_{2} D u_{2} \exp \left\{i \int d ^ { 2 } x \left[\bar{u}_{1}\left(E-H_{0}+i 0^{+}\right) u_{1}-\bar{u}_{2}\left(E-H_{0}+i 0^{-}\right) u_{2}\right.\right. \\
& \left.\left.+V\left(\bar{u}_{1} u_{1}-\bar{u}_{2} u_{2}\right)+\bar{j}_{1} \bar{u}_{1}+\bar{j}_{2} \bar{u}_{2}+j_{1} u_{1}+j_{2} u_{2}\right]\right\}
\end{aligned}
$$

where the fields $u_{1}$ and $u_{2}$ are chosen to be c-numbers. In this way, we have

$$
<G_{+}\left(\vec{x}, \vec{x}^{\prime} ; E\right) G_{-}\left(\vec{x}, \vec{x}^{\prime} ; E\right)>=\int D V P[V] \frac{1}{Z} \frac{\delta^{4} Z}{\delta \vec{j}_{1}(\vec{x}) \delta j_{1}\left(\vec{x}^{\prime}\right) \delta \vec{j}_{2}(\vec{x}) \delta j_{2}\left(\vec{x}^{\prime}\right)}
$$

with the derivatives evaluated at $\bar{j}, j=0$ and $P[V]$ representing the probability distribution function of the random potential $V$. We will be concentrated on the gaussian case, given by $<V(\vec{x}) V\left(\vec{x}^{\prime}\right)>=\lambda \delta^{2}\left(\vec{x}-\vec{x}^{\prime}\right)$. An interesting trick allows us to write $Z^{-1}[0,0,0,0]$, which appears in (2.4), with the same functional form as (2.3), replacing the boson fields $u_{1}$ and $u_{2}$ by fermionic ones, $v_{1}$ and $v_{2}$. Therefore, in a compact notation, all the information about localization is contained in the generating functional (we will not write the currents $\bar{j}$ and $j$ to avoid cumbersome algebra)

$$
Z=\int D V P[V] D \bar{\varphi} D \varphi \exp \left\{i \int d^{2} x\left[\bar{\varphi}\left(\left(E-H_{0}\right) \mathbf{A}+i 0^{+}\right) \varphi+V \bar{\varphi} \mathbf{A} \varphi\right]\right\}
$$

where,

$$
\begin{gathered}
\varphi=\left(\begin{array}{l}
u \\
v
\end{array}\right) \\
u=\left(\begin{array}{l}
u_{1} \\
u_{2}
\end{array}\right), v=\left(\begin{array}{l}
v_{1} \\
v_{2}
\end{array}\right)
\end{gathered}
$$

and the matrix $\mathbf{A}$, acting on the components $u$ and $v$ of $\varphi$, is given by

$$
\left(\begin{array}{cc}
\sigma_{3} & 0 \\
0 & \sigma_{3}
\end{array}\right)
$$

where $\sigma_{3}$ is a Pauli matrix, which acts on the components of $u$ and $v$. In the case of white noise random potentials, the integration over $V$ in (2.5) yields

$$
Z=\int D \bar{\varphi} D \varphi \exp \left\{i \int d^{2} x\left[\bar{\varphi}\left(\left(E-H_{0}\right) \mathbf{A}+i 0^{+}\right) \varphi+i \lambda(\bar{\varphi} \mathbf{A} \varphi)^{2}\right]\right\}
$$

In order to study the quantum Hall effect, we may consider the fields $\varphi$ and $\bar{\varphi}$ as expressed in terms of states belonging only to a specific non perturbed Landau level and replace $H_{0}$ by 
$E_{n}=(n+1 / 2) \hbar \omega_{c}$, where $\omega_{c}$ is the cyclotron frequency. This representation of $\varphi$ corresponds to the assumption of the absence of Landau level mixing. Let us also suppose that when $E \rightarrow E_{n}$, the localization length obtained from (2.9) has the form $\xi\left(E-E_{n}\right) \sim\left|E-E_{n}\right|^{-\nu}$.

The renormalization group computation that will be performed, is a way to find, starting from $Z$, a transformed generating functional

$\tilde{Z}=\int D \bar{\varphi} D \varphi \exp \left\{i \int d^{2} x\left[\bar{\varphi}\left(s\left(E-E_{n}, \lambda\right) \mathbf{A}+i 0^{+}\right) \varphi+i t\left(E-E_{n}, \lambda\right)(\bar{\varphi} \mathbf{A} \varphi)^{2}\right]\right\}$,

where $s\left(E-E_{n}, \lambda\right)$ and $t\left(E-E_{n}, \lambda\right)$ are exactly known, with $s\left(E-E_{n}, \lambda\right) \rightarrow 0$ as $\left(E-E_{n}\right) \rightarrow$ 0 . We assume now that the localization length derived from $\tilde{Z}$ is $\tilde{\xi}=\frac{1}{2} \xi\left(E-E_{n}\right)$.

Since the number of Grassmann and c-number fields in (2.10) is the same, any identical transformation of both fields will produce a unit Jacobian. In particular, we can redefine the field $\varphi$ by

$$
\varphi \rightarrow\left(\frac{\lambda}{t}\right)^{\frac{1}{4}} \varphi
$$

Performing this transformation in $\tilde{Z}$, we get

$$
\tilde{Z}=\int D \bar{\varphi} D \varphi \exp \left\{i \int d^{2} x\left[\bar{\varphi}\left(s\left(E-E_{n}, \lambda\right)\left(\frac{\lambda}{t}\right)^{\frac{1}{2}} \mathbf{A}+i 0^{+}\right) \varphi+i \lambda(\bar{\varphi} \mathbf{A} \varphi)^{2}\right]\right\} \text {. }
$$

Comparing (2.9) with (2.12) and using $\tilde{\xi}=\frac{1}{2} \xi$ we have

$$
\xi\left(s\left(E-E_{n}, \lambda\right)(\lambda / t)^{\frac{1}{2}}\right)=\frac{1}{2} \xi\left(E-E_{n}\right) .
$$

When $E \rightarrow E_{n}$, we may write $s\left(E-E_{n}, \lambda\right)=s^{\prime}(0, \lambda)\left(E-E_{n}\right)$. Substituting the scaling law for $\xi$ in (2.13) we obtain

$$
\left(s^{\prime}(0, \lambda)\left(E-E_{n}\right)(\lambda / t)^{\frac{1}{2}}\right)^{-\nu}=\frac{1}{2}\left(E-E_{n}\right)^{-\nu}
$$

and, therefore,

$$
\nu=\frac{\ln 2}{\ln \left(s^{\prime}(0, \lambda)(\lambda / t)^{\frac{1}{2}}\right)} .
$$

This method for computing $\nu$ is reminiscent from the Kadanoff's block spin renormalization group [14]. In fact, as we will see, the similarity to that method is even deeper than it may be realized from the previous arguments. 


\section{RENORMALIZATION GROUP ANALYSIS}

Let us study the disordered two-dimensional electron gas under the influence of a magnetic field $B$, as defined on a cylinder of radius $L / 2 \pi$, where eventually $L \rightarrow \infty$. In other words, this means that the wavefunctions of the system must satisfy the periodic boundary condition $\psi(x, y)=\psi(x+L, y)$. In the absence of disorder, we may use the Landau gauge, $\vec{A}=B(-y, 0)$, to get the cylinder eigenfunctions of the $n^{\text {th }}$ Landau level,

$$
\psi_{n m}(x, y)=\frac{1}{L^{1 / 2}} H_{n}\left(\left(y-\ell^{2} m h\right) / \ell\right) \exp \left(-\left(y-\ell^{2} m h\right)^{2} / 2 \ell^{2}\right) \exp (i m h x)
$$

where $m=0, \pm 1, \ldots, h \equiv 2 \pi / L, \ell=(c / e B)^{1 / 2}$ is the magnetic length and $H_{n}(x)$ is the Hermite polynomial of order $n$. We can write, thus,

$$
\varphi(x, y)=\left(\frac{h}{2 \pi}\right)^{1 / 2} \sum_{m} H_{n}\left(\left(y-\ell^{2} m h\right) / \ell\right) \exp \left(-\left(y-\ell^{2} m h\right)^{2} / 2 \ell^{2}\right) \exp (i m h x) a_{m h},
$$

with $\bar{\varphi}(x, y)$ analogously defined in terms of $\bar{a}_{m h}$. The fact that the above fields $a_{m h}$ and $\bar{a}_{m h}$ depend on a single variable $m$, reflects the well-known mapping between the quantum Hall effect defined on the cylinder and a one-dimensional fermion system. In particular, the inverse localization length, previously expressed by (2.1), may be rewritten in the onedimensional formalism as

$$
\xi^{-1}(E)=-\frac{1}{2} \lim _{\left|k-k^{\prime}\right| \rightarrow \infty} \frac{\ln <\left(\bar{a}_{k} \mathbf{P}_{+} a_{k^{\prime}}\right)\left(\bar{a}_{k^{\prime}} \mathbf{P}_{-} a_{k}\right)>}{\left|k-k^{\prime}\right|},
$$

where the $4 \times 4$ matrices $\mathbf{P}_{+}$and $\mathbf{P}_{-}$,

$$
\mathbf{P}_{ \pm}=\frac{1}{2}\left(\begin{array}{cc}
I \pm \sigma_{3} & 0 \\
0 & 0
\end{array}\right)
$$

are the projectors which single out the retarded and advanced c-number components of $a_{k}$, respectively.

Using the above expansion of $\varphi(x, y)$, we can express the generating functional (2.9) as

$$
\begin{aligned}
& Z=\int \prod_{m} D \bar{a}_{m h} D a_{m h} \exp \left\{i \sum_{m} \bar{a}_{m h}\left(\left(E-E_{n}\right) \mathbf{A}+i 0^{+}\right) a_{m h}\right. \\
& \left.-\frac{\lambda h}{2 \pi} \sum_{m_{1}, m_{2}, m_{3}} g_{n}\left(\left(m_{1}-m_{2}\right) h,\left(m_{3}-m_{2}\right) h\right)\left(\bar{a}_{m_{1} h} \mathbf{A} a_{m_{2} h}\right)\left(\bar{a}_{m_{3} h} \mathbf{A} a_{\left(m_{1}-m_{2}+m_{3}\right) h}\right)\right\},
\end{aligned}
$$


where

$$
\begin{aligned}
& g_{n}\left(\left(m_{1}-m_{2}\right) h,\left(m_{3}-m_{2}\right) h\right)= \\
& \int d y H_{n}\left(\left(y-\ell^{2} m_{1} h\right) / \ell\right) \exp \left(-\left(y-\ell^{2} m_{1} h\right)^{2} / 2 \ell^{2}\right) H_{n}\left(\left(y-\ell^{2} m_{2} h\right) / \ell\right) \cdot \\
& \cdot \exp \left(-\left(y-\ell^{2} m_{2} h\right)^{2} / 2 \ell^{2}\right) H_{n}\left(\left(y-\ell^{2} m_{3} h\right) / \ell\right) \exp \left(-\left(y-\ell^{2} m_{3} h\right)^{2} / 2 \ell^{2}\right) \cdot \\
& \cdot H_{n}\left(\left(y-\ell^{2}\left(m_{1}-m_{2}+m_{3}\right) h\right) / \ell\right) \exp \left(-\left(y-\ell^{2}\left(m_{1}-m_{2}+m_{3}\right) h\right)^{2} / 2 \ell^{2}\right) .
\end{aligned}
$$

The continuum limit, $h \rightarrow 0$, is, as usual, performed by means of the replacements

$$
\begin{aligned}
& m h \rightarrow k \\
& \sum_{m} \rightarrow \frac{1}{h} \int d k \\
& a_{m h} \rightarrow h^{1 / 2} a_{k} .
\end{aligned}
$$

The continuum definition of $(3.5)$ is, then,

$$
\begin{gathered}
Z=\int D \bar{a}_{k} D a_{k} \exp \left[i \int d k \bar{a}_{k}\left(\left(E-E_{n}\right) \mathbf{A}+i 0^{+}\right) a_{k}\right. \\
\left.-(\lambda / 2 \pi) \int d k_{1} d k_{2} d k_{3} g_{n}\left(k_{1}-k_{2}, k_{3}-k_{2}\right)\left(\bar{a}_{k_{1}} \mathbf{A} a_{k_{2}}\right)\left(\bar{a}_{k_{3}} \mathbf{A} a_{\left(k_{1}-k_{2}+k_{3}\right)}\right)\right] .
\end{gathered}
$$

The generating functional (3.5) describes the dynamics of a multicomponent field defined on a one-dimensional discrete lattice. The first step of the renormalization group transformation consists of the definition of the set of fields $a_{(+, m h)} \equiv a_{2 m h}$ and $a_{(-, m h)} \equiv a_{(2 m+1) h}$, related to the even and odd sites of the lattice, respectively. The point here is that the localization length derived from $a_{(+, m h)}$ or $a_{(-, m h)}$ is precisely $1 / 2$ of the localization length associated to $a_{m h}$, as may be seen from relation (3.3). Expressing now the generating functional (3.5) in terms of $a_{(+, m h)}$ and $a_{(-, m h)}$, we get

$$
Z=\int \prod_{m} D \bar{a}_{(+, m h)} D a_{(+, m h)} D \bar{a}_{(-, m h)} D a_{(-, m h)} \exp \left\{i \sum_{m} S_{m}^{(2)}-\frac{\lambda h}{2 \pi} \sum_{m_{1}, m_{2}, m_{3}} S_{\left(m_{1}, m_{2}, m_{3}\right)}^{(4)}\right\}
$$

with

$$
S_{m}^{(2)}=\bar{a}_{(+, m h)}\left(\left(E-E_{n}\right) \mathbf{A}+i 0^{+}\right) a_{(+, m h)}+\bar{a}_{(-, m h)}\left(\left(E-E_{n}\right) \mathbf{A}+i 0^{+}\right) a_{(-, m h)}
$$


and

$$
\begin{aligned}
& S_{\left(m_{1}, m_{2}, m_{3}\right)}^{(4)}=\bar{a}_{\left(+, m_{1} h\right)} \mathbf{A} a_{\left(+, m_{2} h\right)}\left[g_{n}\left(2\left(m_{1}-m_{2}\right) h, 2\left(m_{3}-m_{2}\right) h\right) \bar{a}_{\left(+, m_{3} h\right)} \mathbf{A} a_{\left(+,\left(m_{1}-m_{2}+m_{3}\right) h\right)}\right. \\
& \left.+g_{n}\left(2\left(m_{1}-m_{2}\right) h, 2\left(m_{3}-m_{2}\right) h+h\right) \bar{a}_{\left(-, m_{3} h\right)} \mathbf{A} a_{\left(-,\left(m_{1}-m_{2}+m_{3}\right) h\right)}\right]+\bar{a}_{\left(+, m_{1} h\right)} \mathbf{A} a_{\left(-, m_{2} h\right)} . \\
& \cdot\left[g_{n}\left(2\left(m_{1}-m_{2}\right) h-h, 2\left(m_{3}-m_{2}\right) h-h\right) \bar{a}_{\left(+, m_{3} h\right)} \mathbf{A} a_{\left(-,\left(m_{1}-m_{2}+m_{3}-1\right) h\right)}\right. \\
& \left.+g_{n}\left(2\left(m_{1}-m_{2}\right) h-h, 2\left(m_{3}-m_{2}\right) h\right) \bar{a}_{\left(-, m_{3} h\right)} \mathbf{A} a_{\left(+,\left(m_{1}-m_{2}+m_{3}\right) h\right)}\right] \\
& +\bar{a}_{\left(-, m_{1} h\right)} \mathbf{A} a_{\left(+, m_{2} h\right)}\left[g_{n}\left(2\left(m_{1}-m_{2}\right) h+h, 2\left(m_{3}-m_{2}\right) h\right) \bar{a}_{\left(+, m_{3} h\right)} \mathbf{A} a_{\left(-,\left(m_{1}-m_{2}+m_{3}\right) h\right)}\right. \\
& \left.+g_{n}\left(2\left(m_{1}-m_{2}\right) h+h, 2\left(m_{3}-m_{2}\right) h+h\right) \bar{a}_{\left(-, m_{3} h\right)} \mathbf{A} a_{\left(+,\left(m_{1}-m_{2}+m_{3}+1\right) h\right)}\right] \\
& +\bar{a}_{\left(-, m_{1} h\right)} \mathbf{A} a_{\left(-, m_{2} h\right)}\left[g_{n}\left(2\left(m_{1}-m_{2}\right) h, 2\left(m_{3}-m_{2}\right) h+h\right) \bar{a}_{\left(+, m_{3} h\right)} \mathbf{A} a_{\left(+,\left(m_{1}-m_{2}+m_{3}\right) h\right)}\right. \\
& g_{n}\left(2\left(m_{1}-m_{2}\right) h, 2\left(m_{3}-m_{2}\right) h\right) \bar{a}_{\left(-, m_{3} h\right)} \mathbf{A} a_{\left.\left(-,\left(m_{1}-m_{2}+m_{3}\right) h\right)\right]} .
\end{aligned}
$$

We would like to find, in view of the arguments presented in section II, a renormalized generating functional, related to anyone of the fields $a_{(+, m h)}$ or $a_{(-, m h)}$, which has the same structure as (3.5). In order to do that, let us first note that there are in $S^{(4)}$ some coefficients and fields which differ from the others only by terms of the order of $h$, when $h \rightarrow 0$. We may, therefore, perform an expansion of (3.13) up to $\mathcal{O}(h)$. It is useful, for this purpose, to define

$$
\begin{aligned}
& g_{n}^{(1)}(x, y)=\frac{\partial}{\partial x} g_{n}(x, y) \\
& g_{n}^{(2)}(x, y)=\frac{\partial}{\partial y} g_{n}(x, y)
\end{aligned}
$$

and also

$$
\begin{array}{r}
\Phi_{m h}=\left(\begin{array}{c}
a_{(+, m h)} \\
a_{(-, m h)}
\end{array}\right) \\
\delta \Phi_{m h}=\Phi_{m h}-\Phi_{(m-1) h} .
\end{array}
$$

The result of the expansion gives, after straightforward computations,

$$
Z=\int \prod_{m} D \bar{\Phi}_{m h} D \Phi_{m h} \exp \{i S+i \delta S\}
$$

where 


$$
\begin{aligned}
& S=\sum_{m} \bar{\Phi}_{m h}\left(\left(E-E_{n}\right) \mathbf{A}+i 0^{+}\right) \Phi_{m h}+\frac{i \lambda h}{2 \pi} \sum_{\{m\}} g_{n} \cdot\left[\left(\bar{\Phi}_{m_{1} h} \mathbf{A} \Phi_{m_{2} h}\right)\right. \\
& \left.\cdot\left(\Phi_{m_{3} h} \mathbf{A} \Phi_{\left(m_{1}-m_{2}+m_{3}\right) h}\right)+\left(\bar{\Phi}_{m_{1} h} \mathbf{A} \sigma_{1} \Phi_{m_{2} h}\right)\left(\bar{\Phi}_{m_{3} h} \mathbf{A} \sigma_{1} \Phi_{\left(m_{1}-m_{2}+m_{3}\right) h}\right)\right]
\end{aligned}
$$

and

$$
\begin{aligned}
& \delta S=i \lambda \sum_{m_{1}, m_{2}, m_{3}}\left\{\frac{h^{2}}{2 \pi} g_{n}^{(2)} \cdot\left(\bar{\Phi}_{m_{1} h} \frac{\left(1+\sigma_{3}\right)}{2} \mathbf{A} \Phi_{m_{2} h}\right)\left(\bar{\Phi}_{m_{3} h} \frac{\left(1-\sigma_{3}\right)}{2} \mathbf{A} \Phi_{\left(m_{1}-m_{2}+m_{3}\right) h}\right)\right. \\
& -\frac{h^{2}}{2 \pi}\left(g_{n}^{(1)}+g_{n}^{(2)}\right) \cdot\left(\bar{\Phi}_{m_{1} h} \frac{\left(\sigma_{1}+i \sigma_{2}\right)}{2} \mathbf{A} \Phi_{m_{2} h}\right)\left(\bar{\Phi}_{m_{3} h} \frac{\left(\sigma_{1}+i \sigma_{2}\right)}{2} \mathbf{A} \Phi_{\left(m_{1}-m_{2}+m_{3}\right) h}\right) \\
& -\frac{h^{2}}{4 \pi} g_{n}^{(1)} \cdot\left[\left(\bar{\Phi}_{m_{1} h} \frac{\left(\sigma_{1}+i \sigma_{2}\right)}{2} \mathbf{A} \Phi_{m_{2} h}\right)\left(\bar{\Phi}_{m_{3} h} \frac{\left(\sigma_{1}-i \sigma_{2}\right)}{2} \mathbf{A} \Phi_{\left(m_{1}-m_{2}+m_{3}\right) h}\right)+(+\longleftrightarrow-)\right] \\
& \left.-\frac{h}{2 \pi} g_{n} \cdot\left(\bar{\Phi}_{m_{1} h} \frac{\left(\sigma_{1}+i \sigma_{2}\right)}{2} \mathbf{A} \Phi_{m_{2} h}\right)\left(\bar{\Phi}_{m_{3} h} \frac{\left(\sigma_{1}+i \sigma_{2}\right)}{2} \mathbf{A} \delta \Phi_{\left(m_{1}-m_{2}+m_{3}\right) h}\right)+c . c\right\} .
\end{aligned}
$$

In $(3.19)$ and $(\overline{3.20})$ the functions $g_{n}, g_{n}^{(1)}$ and $g_{n}^{(2)}$ are implicitly defined on the point $(x, y)=$ $\left(2\left(m_{1}-m_{2}\right) h, 2\left(m_{3}-m_{2}\right) h\right)$. In these equations, the Pauli matrices $\sigma_{1}, \sigma_{2}$ and $\sigma_{3}$ act on the components $a_{(+, m h)}$ and $a_{(-, m h)}$ of $\Phi_{m h}$.

Considering that near the "critical point", $E=E_{n}$, the field $\Phi_{m h}$ will not fluctuate considerably in a certain range of sites of the one-dimensional lattice, we may write $\delta \Phi_{m h} \rightarrow$ $h^{3 / 2} \frac{\partial}{\partial k} \Phi_{k}$ as $h \rightarrow 0$, and it is immediately seen that $\delta S$ vanishes in the continuum limit. We will study, thus, only the action $S$, given by (3.19), in the next computations. It is interesting to note, however, that there is an $O(2)$ symmetry in the problem, expressing the invariance of $S$ under the mapping $\Phi_{m h} \rightarrow \exp \left(i \theta \sigma_{1}\right) \Phi_{m h}$, which is explicitly broken by $\delta S$.

We can use a $S U(2)$ transformation, given by $U^{+} \sigma_{1} U=\sigma_{3}, \Phi_{m h} \rightarrow U \Phi_{m h}$ and $\bar{\Phi}_{m h} \rightarrow$ $\bar{\Phi}_{m h} U^{+}$, to find, from (3.19), $Z=Z_{+} Z_{-}$, where

$$
\begin{aligned}
& Z_{+}=\int \prod_{m} D \bar{a}_{(+, m h)} D a_{(+, m h)} \exp \left\{i \sum_{m} \bar{a}_{(+, m h)}\left(\left(E-E_{n}\right) \mathbf{A}+i 0^{+}\right) a_{(+, m h)}-2 \frac{\lambda h}{2 \pi} .\right. \\
& \left.\cdot \sum_{m_{1}, m_{2}, m_{3}} g_{n}\left(2\left(m_{1}-m_{2}\right) h, 2\left(m_{3}-m_{2}\right) h\right)\left(\bar{a}_{\left(+, m_{1} h\right)} \mathbf{A} a_{\left(+, m_{2} h\right)}\right)\left(\bar{a}_{\left(+, m_{3} h\right)} \mathbf{A} a_{\left(+,\left(m_{1}-m_{2}+m_{3}\right) h\right)}\right)\right\}
\end{aligned}
$$

and similarly for $Z_{-}$. We will prove now that the above $S U(2)$ rotation does not modify the localization length associated to each one of the components of $\Phi_{m h}$ and so (3.21) gives 
us a theory with a localization length which is $1 / 2$ of that one derived from $Z$, defined by (3.5). In order to understand it, let us note that, according to (3.3), we have to study how the correlation function

$$
<\left(\bar{a}_{(+, m h)} \mathbf{P}_{+} a_{\left(+, m^{\prime} h\right)}\right)\left(\bar{a}_{\left(+, m^{\prime} h\right)} \mathbf{P}_{-} a_{(+, m h)}\right)>
$$

is modified when the $S U(2)$ rotation,

$$
a_{(+, m h)} \rightarrow \frac{1}{\sqrt{2}}\left(a_{(+, m h)}+a_{(-, m h)}\right)
$$

is performed. Therefore, substituting (3.23) in (3.22), we get

$$
\begin{aligned}
& \frac{1}{4}<\left[\left(\bar{a}_{(+, m h)}+\bar{a}_{(-, m h)}\right) \mathbf{P}_{+}\left(a_{\left(+, m^{\prime} h\right)}+a_{\left(-, m^{\prime} h\right)}\right)\right]\left[\left(\bar{a}_{\left(+, m^{\prime} h\right)}+\bar{a}_{\left(-, m^{\prime} h\right)}\right) \mathbf{P}_{-} .\right. \\
& \left.\cdot\left(a_{(+, m h)}+a_{(-, m h)}\right)\right]>.
\end{aligned}
$$

The above correlation function must be evaluated in the theory defined by $Z=Z_{+} Z_{-}$. The factorization of $Z$ implies that (3.24) is exactly

$$
\frac{1}{2}\left\{<\left(\bar{a}_{(+, m h)} \mathbf{P}_{+} a_{\left(+, m^{\prime} h\right)}\right)\left(\bar{a}_{\left(+, m^{\prime} h\right)} \mathbf{P}_{-} a_{(+, m h)}\right)>+\left|<\left(\bar{a}_{(+, m h)} \mathbf{P}_{+} a_{\left(+, m^{\prime} h\right)}\right)>\right|^{2}\right\}
$$

where we used, between (3.24) and (3.25), the fact that the fields $a_{(+, m h)}$ and $a_{(-, m h)}$ have identical correlation functions. Now, according to the general result for the averaged Green's functions, stating that $\left|<G\left(k, k^{\prime} ; E\right)>\right|^{2}$ vanishes faster than $<\left|G\left(k, k^{\prime} ; E\right)\right|^{2}>$ when $\left|k-k^{\prime}\right| \rightarrow \infty$, we can see that the second term in (3.25) may be neglected, in order to compute the localization length. We find, then, that the only effect of the $S U(2)$ transformation is a factor of $1 / 2$ multiplying the original correlation function (3.22), which, from (3.3), does not modify the value obtained for $\xi(E)$.

In the continuum limit, $Z_{+}$is written as

$$
\begin{gathered}
Z_{+}=\int D \bar{a}_{(+, k)} D a_{(+, k)} \exp \left[i \int d k \bar{a}_{(+, k)}\left(\left(E-E_{n}\right) \mathbf{A}+i 0^{+}\right) a_{(+, k)}\right. \\
\left.-2 \frac{\lambda}{2 \pi} \int d k_{1} d k_{2} d k_{3} g_{n}\left(2\left(k_{1}-k_{2}\right), 2\left(k_{3}-k_{2}\right)\right)\left(\bar{a}_{\left(+, k_{1}\right)} \mathbf{A} a_{\left(+, k_{2}\right)}\right)\left(\bar{a}_{\left(+, k_{3}\right)} \mathbf{A} a_{\left(+, k_{1}-k_{2}+k_{3}\right)}\right)\right] .
\end{gathered}
$$


We can, in the same way, iterate $Z_{+}$to find a new generating functional $Z_{++}$and so on. After $N$ iterations of $Z$, we will have

$$
\begin{gathered}
Z^{(N)}=\int D \bar{a}_{k} D a_{k} \exp \left[i \int d k \bar{a}_{k}\left(\left(E-E_{n}\right) \mathbf{A}+i 0^{+}\right) a_{k}\right. \\
\left.-2^{N} \frac{\lambda}{2 \pi} \int d k_{1} d k_{2} d k_{3} g_{n}\left(2^{N}\left(k_{1}-k_{2}\right), 2^{N}\left(k_{3}-k_{2}\right)\right)\left(\bar{a}_{k_{1}} \mathbf{A} a_{k_{2}}\right)\left(\bar{a}_{k_{3}} \mathbf{A} a_{\left(k_{1}-k_{2}+k_{3}\right)}\right)\right] .
\end{gathered}
$$

One may observe that the above renormalized generating functional can be obtained directly from $Z$, eq. (3.10), through the mapping $a_{k} \rightarrow \frac{1}{2^{N / 2}} a_{\left(k / 2^{N}\right)}$. Regarding $g_{n}$ as a distribution (see the appendix A), we find, from (3.6),

$$
\lim _{N \rightarrow \infty} g_{n}\left(2^{N}\left(k_{1}-k_{2}\right), 2^{N}\left(k_{3}-k_{2}\right)\right)=\frac{C_{n}}{2^{2 N}} \delta\left(k_{1}-k_{2}\right) \delta\left(k_{3}-k_{2}\right)
$$

where it is unimportant to know the exact value of $C_{n}$. Substituting (3.28) in (3.27), we get the asymptotic form of $Z^{(N)}$,

$$
Z^{(N)}=\int D \bar{a}_{k} D a_{k} \exp \left[i \int d k \bar{a}_{k}\left(\left(E-E_{n}\right) \mathbf{A}+i 0^{+}\right) a_{k}-\frac{\lambda}{2 \pi} \frac{C_{n}}{2^{N}} \int d k\left(\bar{a}_{k} \mathbf{A} a_{k}\right)^{2}\right],
$$

where the term proportional to $\lambda$ has to be considered as a point splitted product of fields. The above definition of the asymptotic form of $Z^{(N)}$ is equivalent to neglecting irrelevant terms of subleading order, in the framework of the renormalization group transformation we have been studying. More precisely, let us consider a power expansion of the product of four fields

$$
\begin{aligned}
& \left(\bar{a}_{k_{1}} \mathbf{A} a_{k_{2}}\right)\left(\bar{a}_{k_{3}} \mathbf{A} a_{\left(k_{1}-k_{2}+k_{3}\right)}\right)=\sum_{n_{1}, n_{2}, n_{3}=0}^{\infty} c\left(n_{1}, n_{2}, n_{3}\right)\left(k_{2}-k_{1}\right)^{n_{1}}\left(k_{3}-k_{1}\right)^{n_{2}}\left(k_{3}-k_{2}\right)^{n_{3}} . \\
& \cdot\left(\bar{a}_{k_{1}} \mathbf{A}\left(\frac{\partial^{n_{1}}}{\partial k_{1}^{n_{1}}} a_{k_{1}}\right)\right)\left(\left(\frac{\partial^{n_{2}}}{\partial k_{1}^{n_{2}}} \bar{a}_{k_{1}}\right) \mathbf{A}\left(\frac{\partial^{n_{3}}}{\partial k_{1}^{n_{3}}} a_{k_{1}}\right)\right) .
\end{aligned}
$$

In $(3.30)$ there is a sequence of products of operators, defined at the same point $k_{1}$. This may be regularized through the point splitting procedure, which consists, if one wants to analyze the generating functional $Z^{(N)}$, of chosing a scale $\tau \ll 1 /\left(2^{N} \ell\right)$ and writing the product of four generic operators $O_{1}(k), O_{2}(k), O_{3}(k)$ and $O_{4}(k)$ as 


$$
O_{1}(k) O_{2}(k) O_{3}(k) O_{4}(k)=\frac{1}{\tau^{2}} \int_{-\tau / 2}^{\tau / 2} \int_{-\tau / 2}^{\tau / 2} d p d q O_{1}(k) O_{2}(k+p) O_{3}(k+p+q) O_{4}(k+q) .
$$

The limit $\tau \rightarrow 0$ is performed after the computation physical quantities. Substituting (3.30) in (3.27) we will have a sum of terms with coefficients given by

$$
2^{N} \frac{\lambda}{2 \pi} \int d p d q g_{n}\left(2^{N} p, 2^{N} q\right) c\left(n_{1}, n_{2}, n_{3}\right) p^{n_{1}}(p+q)^{n_{2}} q^{n_{3}} \sim \frac{\lambda}{2 \pi} \frac{c\left(n_{1}, n_{2}, n_{3}\right)}{2^{N\left(n_{1}+n_{2}+n_{3}+1\right)}} .
$$

In the language of the renormalization group theory, (3.32) means that we have an infinite set of irrelevant couplings. The asymptotic form (3.29) includes the effects of only the leading term in this expansion, corresponding to $n_{1}, n_{2}, n_{3}=0$. We see, therefore, from (3.29), that $Z^{(N)} \rightarrow Z^{(N+1)}$ is obtained from $\left(E-E_{n}\right) \rightarrow\left(E-E_{n}\right)$ and $\lambda \rightarrow \lambda / 2$. According to the discussion of section II, the functions $s$ and $t$ for this mapping are $s(x, y)=x$ and $t(x, y)=y / 2$. Applying now eq. (2.15) we get $\nu=2$.

\section{CONCLUSION}

Using the two basic assumptions of the existence of a localization length scaling law and the absence of Landau level mixing, we were able to find, neglecting irrelevant couplings of subleading order, the value $\nu=2$ for the localization length exponent, in all Landau levels. The method of computation is based on an exact sequence of renormalization group transformations. The fundamental ingredient in the analysis is that after each transformation of the generating functional of Green's functions, we obtain a theory which gives $1 / 2$ of the previous localization length.

It is possible that the neglected terms (the one containing $\delta \Phi_{m h}$ in (3.20) and the terms with coefficients expressed by (3.32), for $n_{1}+n_{2}+n_{3} \geq 1$ ) have a participation in the evaluation of $\nu$, similar to what happens when dangerous irrelevant variables come into play in the statistical mechanics of phase transitions. The subleading terms could account for the fact that there is already some numerical evidence establishing that $\nu \sim 2.3$ [9]. Related to it, we advance that $\nu=2$ may be associated to a possible intermediate scaling region 
in the crossover between the localization length exponent obtained via classical percolation theory 15, $\nu=4 / 3$, and $\nu \sim 2.3$, occuring as the correlation length of the disordered potential vanishes. A physical interpretation of this intermediate region would be that the neglected couplings, containing derivatives, are not expected to contribute when the system is characterized by disordered potentials correlated in very large scales.

However, even if $\nu=2$ as the true asymptotic exponent seems to be a remote possibility, we cannot rule out some conjectures originated from the fact that the sizes used in numerical computations are really small, and the range of energies limited by the requirement of numerical precision. At least, we can propose two possibilities, both based essentially on the idea of crossover: 1) there may be a crossover from $\nu \sim 2.3$ to $\nu=2$ as the energy approaches the center of a broadened Landau level; 2) as may be inferred from section III, finite size effects break a $O(2)^{N}$ symmetry between the $2^{N}$ fields introduced after $N$ steps of the renormalization group iteration. It is possible, then, to have a modification of the critical exponents, reflecting the existence of symmetries which are exact only in the thermodynamic limit. In this situation, one would expect a crossover to $\nu=2$, at fixed energy, as the thermodynamic limit is reached. A related observation is that in conventional Anderson localization, a crossover may be induced through the breaking of spin and timereversal symmetries [16].

It would be interesting to apply the present formalism to other close problems, as, for instance, the analysis of alternative definitions of disordered potentials and the computation of conductivity properties at the metal-insulator transition.

\section{ACKNOWLEDGMENTS}

I would like to thank R. Bhatt, A. Ludwig and M. Moriconi for interesting discussions. Also, I would like to thank a referee for calling my atention to ref. [4]. This work was supported by $\mathrm{CNPq}($ Brazil). 


\section{APPENDIX A: DERIVATION OF (3.28)}

In terms of the generating function of Hermite polynomials, $f(w)=\exp \left(-w^{2}+2 w s\right)$, we have, according to (3.19),

$$
\begin{aligned}
& g_{n}\left(2^{N}\left(k_{1}-k_{2}\right), 2^{N}\left(k_{3}-k_{2}\right)\right)=\frac{\partial^{n}}{\partial w_{1}^{n}} \frac{\partial^{n}}{\partial w_{2}^{n}} \frac{\partial^{n}}{\partial w_{3}^{n}} \frac{\partial^{n}}{\partial w_{4}^{n}} \int d y \exp \left[-\left(w_{1}^{2}+w_{2}^{2}+w_{3}^{2}+w_{4}^{2}\right)\right. \\
& \left.+2\left(w_{1} s_{1}+w_{2} s_{2}+w_{3} s_{3}+w_{4} s_{4}\right)-\left(s_{1}^{2}+s_{2}^{2}+s_{3}^{2}+s_{4}^{2}\right) / 2\right]
\end{aligned}
$$

where

$$
\begin{aligned}
& s_{1}=\left(y-\ell^{2} 2^{N} k_{1}\right) / \ell \\
& s_{2}=\left(y-\ell^{2} 2^{N} k_{2}\right) / \ell \\
& s_{3}=\left(y-\ell^{2} 2^{N} k_{3}\right) / \ell \\
& s_{4}=\left(y-\ell^{2} 2^{N}\left(k_{1}-k_{2}+k_{3}\right)\right) / \ell
\end{aligned}
$$

and the derivatives are taken at $w_{1}, w_{2}, w_{3}, w_{4}=0$. The integration over $y$ in (A1) yields

$$
\begin{aligned}
& g_{n}=\sqrt{\frac{\pi}{2}} \ell \exp \left\{-2^{2 N} \frac{\ell^{2}}{2}\left[\left(k_{1}-k_{2}\right)^{2}+\left(k_{3}-k_{2}\right)^{2}\right]\right\} \frac{\partial^{n}}{\partial w_{1}^{n}} \frac{\partial^{n}}{\partial w_{2}^{n}} \frac{\partial^{n}}{\partial w_{3}^{n}} \frac{\partial^{n}}{\partial w_{4}^{n}} \\
& \exp \left[-\left(w_{1}^{2}+w_{2}^{2}+w_{3}^{2}+w_{4}^{2}\right)+\frac{1}{2}\left(w_{1}+w_{2}+w_{3}+w_{4}\right)^{2}+2^{N} \ell\left(k_{1}+k_{3}\right)\left(w_{1}+w_{2}+w_{3}+w_{4}\right)\right. \\
& \left.-2^{N+1} \ell\left(w_{1} k_{1}+w_{2} k_{2}+w_{3} k_{3}+w_{4}\left(k_{1}-k_{2}+k_{3}\right)\right)\right] .
\end{aligned}
$$

Using, then (in the sense of distribution theory) that $\exp \left(-a x^{2}\right) \sim a^{-1 / 2} \delta(x)$ when $a \rightarrow \infty$, we get, from (A3),

$$
g_{n}=\frac{C_{n}}{2^{2 N}} \delta\left(k_{1}-k_{2}\right) \delta\left(k_{3}-k_{2}\right)
$$

as the leading term when $N \rightarrow \infty$. The coefficient $C_{n}$ is writen as

$$
\begin{aligned}
& C_{n} \sim \frac{\partial^{n}}{\partial w_{1}^{n}} \frac{\partial^{n}}{\partial w_{2}^{n}} \frac{\partial^{n}}{\partial w_{3}^{n}} \frac{\partial^{n}}{\partial w_{4}^{n}} \exp \left[-\left(w_{1}^{2}+w_{2}^{2}+w_{3}^{2}+w_{4}^{2}\right)+\frac{1}{2}\left(w_{1}+w_{2}+w_{3}+w_{4}\right)^{2}\right] \\
& =\int d s\left(H_{n}(s) \exp \left(-\frac{1}{2} s^{2}\right)\right)^{4} .
\end{aligned}
$$




\section{REFERENCES}

[1] K. Von Klitzing, G. Ebert and M. Pepper, Phys. Rev. Lett. 45, 494 (1980).

[2] E. Abrahams, P. W. Anderson, D. C. Licciardello, and T. V. Ramakrishnan, Phys. Rev. Lett. 42, 673 (1979).

[3] S. Hikami, Prog. Theor. Phys. 76, 1210 (1986).

[4] M. Jansen, Int. J. Mod. Phys. B 8, 943 (1994).

[5] G. V. Mil'nikov and I. M. Sokolov, Pis'ma Zh. Eksp. Teor. Fiz. 48, 494 (1988) [JETP Lett. 48, 536 (1988)].

[6] S. Gurvitz, Resonant Scattering on Impurities in the Integer Quantum Hall Effect, TRIPP-94-33, cond-mat@babbage.sissa.it 9406022.

[7] H. Aoki and T. Ando, Phys. Rev. Lett. 54, 831 (1985).

[8] J. T. Chalker and P. D. Coddington, J. Phys. C 21, 2665 (1988).

[9] B. Huckstein and B. Kramer, Phys. Rev. Lett. 64, 1437 (1990).

[10] Y. Huo and R. N. Bhatt, Phys. Rev. Lett. 68, 1375 (1992).

[11] D.-H. Lee, Z. Wang and S. Kivelson, Phys. Rev. Lett. 70, 4130 (1993).

[12] D. Liu and S. Das Sarma, Phys. Rev. B 49, 2677 (1994).

[13] S. Koch, R. J. Haug, K. Von Klitzing, and K. Ploog, Phys. Rev. B 43, 6828 (1991).

[14] L. P. Kadanoff, Physics(N.Y.) 2, 263 (1966).

[15] S. A. Trugman, Phys. Rev. B 27, 7539 (1983).

[16] F. J. Wegner, Nucl. Phys. B270, 1 (1986). 